\title{
Chemistry of Diffuse Clouds and Circumstellar Envelopes
}

\author{
Robert Lucas \\ IRAM, 300 rue de la Piscine, 38406 Saint-Martin-d'Hères, France
}

\begin{abstract}
We present and review recent millimeter-wave observations with interferometers, in particular, with the Plateau de Bure interferometer, that stress the importance of high-frequency resolution for studying the chemistry of diffuse interstellar clouds and of post main-sequence circumstellar envelopes. The future impact of new instruments, such as the Atacama Large Millimeter and submillimeter Array (ALMA), is also investigated.
\end{abstract}

\section{Introduction}

High-resolution spectroscopy in the millimeter and sub-millimeter wavelength domains uses heterodyne reception. In most cases the sensitivity is determined by the collecting areas and by the noise temperatures of the radio receiver frontend parts (mixer, amplifiers). The spectral resolution is determined by the backend part of the receiving systems, which can consist of filter banks, acoustooptical spectrometers, or correlators.

Due to recent, steady progress in digital technology, the cost of correlators has been decreasing, and they are or will be used in most large ground-based instruments (not only interferometers, but also single-dish telescopes). Correlators have the interesting property that the spectral resolution is tunable (generally, however, at the cost of lower total bandwidth).

Spectral correlators have a steadily increasing number of channels, so that in a typical situation the maximum bandwith could soon be determined by the instantaneous front-end bandwith, while the actual spectral resolution is really limited by sensitivity, or by the affordable digital data rate. Spectral correlators are suitable for most observational projects with the possible exception of some spectral surveys, including the search for very narrow lines in the whole front-end bandwidth).

In the millimeter domain, in particular, the spectral resolution is limited by sensitivity, just like the spatial resolution in the case of aperture synthesis. As an example, in the last fifteen years the Plateau de Bure array had two generations of front-ends and four successive correlator systems. The latest one enables full analysis of the available intermediate frequency band and few (if any) projects are limited by the available spectral capability.

Correlators still suffer from a (small) loss of sensitivity due to digitization, but the use of more bits limits this effect. 


\section{Diffuse Clouds Seen in Millimeter-wave Absorption}

Absorption is a powerful tool for detecting low-excitation gas, e.g., molecules in diffuse clouds as has been observed in the optical and UV spectral ranges for more than 60 years. For millimeter-wave absorption, we use different background sources, e.g., quasars and distant galaxies instead of stars. Unfortunately, this makes direct, precise comparison with optical/UV results rather difficult.

The low density makes interpretation easier: many molecules are in in equilibrium with the cosmic background radiation, and thus only one rotational line is enough to measure a good column density. This is not the case, however, for $\mathrm{CO}$, which can be excited at low densities (a few $100 \mathrm{~cm}^{-3}$ ). Due to their larger transition probabilities, high-dipole moment molecules $\left(\mathrm{HCO}^{+}, \mathrm{HCN}\right)$ are easier to detect than low-dipole moment ones (e.g., CO).

It is worthwhile to note that this observing method works with the same sensitivity for gas in distant galaxies: see, for instance, Wiklind (2003).

From an observational point of view, the observations are rather easy, as the background source is point-like and strong enough $(\sim 0.5 \mathrm{Jy})$ to allow selfcalibration of amplitudes and phases. Such observations can make a good use of relatively poor weather conditions. Interferometers advantageously resolve out the emission component if present, for instance, in the $\mathrm{CO}$ lines.

Among the main results is the surprisingly high abundance of molecules like $\mathrm{HCO}^{+}$and $\mathrm{HCN}$ in diffuse clonds (Lucas \& Liszt 1996). There is a remarkable relation between $\mathrm{HCO}^{+}$and $\mathrm{OH}$. The turn on of $\mathrm{CO}$ at $N\left(\mathrm{HCO}^{+}\right) \sim 10^{12} \mathrm{~cm}^{-2}$ is observed, corresponding to the transition from $\mathrm{C}^{+}$to $\mathrm{C}$ and $\mathrm{CO}$ (Liszt \& Lucas 1998). $\mathrm{C}_{2} \mathrm{H}$ is also a very common species; many molecules like $\mathrm{HCN}$, $\mathrm{CN}$, and $\mathrm{HNC}$ have relative abundances comparable to those in dark clouds, with notable exceptions $\left(\mathrm{N}_{2}^{+}, \mathrm{CH}_{3} \mathrm{CN}\right)$. The chemical pathways leading to these high abundances are still far from being understood.

\section{Circumstellar Envelopes}

Circumstellar envelopes (CSEs), which appear around stars of masses $\sim 0.8-8$ $\mathrm{M}_{\odot}$ at the end of their evolution, trace the process through which these stars return heavy elements and dust particles to the interstellar medium. They are the sites of very rich (O- or C-rich) chemistry with complex molecules, which is probed by line emission and dust continuum emission in the radio, sub-mm, infrared. In the inner regions dust forms, is accelerated by radiation pressure, and drives the gas; while in outer regions the interstellar UV radiation strongly drives the chemistry (eventually destroying most molecules). See e.g., Glassgold (1999) and Lucas \& Guélin (1999) for reviews.

Several CSEs have detached CO shells, probably resulting from ancient $(\sim 800$ to $\sim 8000 \mathrm{y}$ ) short (a few 100y) episodes of higher mass-loss. So far only $\mathrm{CO}$ is detected in large detached shells, but $\mathrm{HCN}$ and $\mathrm{CN}$ are detected in U Cam. The outer envelopes of IRC+10216, CRL 2688, NGC7027, and others show multiple shell structures indicating mass loss rate variations (see e.g., Mauron \& Huggins 2000; Fong et al. 2003). 


\section{ALMA Perspectives}

ALMA will support both high angular resolution and high-frequency resolution observations, 0.18 and $0.018 \mathrm{~km} \mathrm{~s}^{-1}$ at $3 \mathrm{~mm}$ and $0.3 \mathrm{~mm}$, respectively. Note that these numbers should be multiplied by 4 for full polarization work. A second generation correlator is under study (but not yet budgeted) for still higher resolution and more frequency channels at the highest resolutions.

Diffuse Clouds With the full-scale ALMA (planned for 2012) the limiting flux to reach 0.01 sensitivity in optical depth for a $1 \mathrm{~km} \mathrm{~s}^{-1}$ wide line will be $\sim 100 \mathrm{mJy}$, compared with $1.5 \mathrm{Jy}$ for the Plateau de Bure array. Several thousands of background sources should be then observable compared to $\sim 40$. One will be able to perform more complete studies of the diffuse gas than is now possible in the optical/UV, such as a large survey of $\mathrm{HCO}^{+}$absorption to study more extensively the structure and dynamics of the diffuse gas, compare diffuse cloud chemistry in various regions of the Galaxy, and complete a line survey in front of a few strong sources. One may also select lines of sight passing through transluscent clouds and even denser clouds, to obtain chemical abundance measurements unbiased by selective collisional excitation, and compare local results with those from nearby external galaxies for which suitable background sources can be found.

Circumstellar envelopes With the high angular spatial resolution of ALMA, one may map and monitor the chemistry in the inner region (about $0.5^{\prime \prime}$ in IRC+10216), where dust forms and gas is accelerated, using as probes CO, $\mathrm{SiO}$, SiS, and HCN. It will be possible to access submillimeter lines of higher excitation since the inner envelopes have higher temperatures and densities. With high sensitivity, one will perform more extensive spectral surveys in more diverse stars and at more stages of evolution. More extensive mapping will help us understand the mass-loss history better and the related ring structure in extended envelopes as well as in detached shells. High angular resolution is needed to resolve the shell structures. Surveys of CSEs will be extended to different regions of the Galaxy and nearby galaxies (e.g., the Magellanic Clouds). For many of these studies comparison with other large instruments such as the VLT, VLTI, Hershel, and new generation space telescopes will be essential.

\section{References}

Glassgold, A. E. 1999, in IAU Symp. 191, Asymptotic Giant Branch Stars, ed. T. Le Bertre, A. Lèbre, \& C. Waelkens (San Francisco: ASP), 337

Fong, D., Meixner, M., \& Shah, R. Y. 2003, ApJ, 582, L39

Liszt, H. S., \& Lucas, R. 1998, A\&A, 339, 561

Lucas, R., \& Guélin, M., in IAU Symp. 191, Asymptotic Giant Branch Stars, ed. T. Le Bertre, A. Lèbre, \& C. Waelkens (San Francisco: ASP), 305

Lucas, R., \& Liszt, H. S. 1996, A\&A, 307, 237

Mauron, N., \& Huggins, P. J. 2000, A\&A, 359, 707

Wiklind, T., This volume, JD 21 